Pacific Journal of Mathematic 


\title{
METRIC AVERAGING IN EUCLIDEAN AND HILBERT SPACES
}

\section{RALPH ALEXANDER}

\begin{abstract}
A number of geometric properties of sets in $l^{2}$ can be measured in terms of maxima and minima of quadratic forms subject to side conditions. A method of metric addition which exploits this fact is investigated. The main objective is to develop a flexible method for attacking geometric extremal problems involving sums of distances or other metric constraints.
\end{abstract}

1. Introduction and principal embedding theorem. The general approach for metric addition, or averaging, in Euclidean and Hilbert spaces has proved useful in the study of a number of geometric extremal problems (See [1], [2].) However, in this earlier work we did not realize that the various averaging processes and inequalities could be presented in a completely unified manner.

Obviously, we can make no claim of total originality since special cases of our results are well-known. In fact in $\S 6$ we point out that an inequality is equivalent to a known result for positive definite matrices. However we do feel that our method, based on ideas related to an embedding Theorem of I. J. Schoenberg [9], possesses scope and flexibility.

The general method exploits the fact that a number of geometric properties of sets in $l^{2}$ can be measured in terms of maxima and minima of quadratic forms subject to linear side conditions.

The logic of the method's application to extremal problems parallels that of Minkowski (or vector) addition. One assumes that an extremal configuration is at hand, and by "adding" suitable copies of the configuration one hopes to determine the nature of the configuration by use of inequalities and other properties which are preserved by the addition process. In $\S 7$ this is illustrated by an example.

Let $(Y, \tau)$ be a compact topological space which can be homeomorphically embedded in the classical Hilbert space $l^{2}$, and let $T$ be a family of homeomorphisms from $Y$ into $l^{2}$ such that the images $Y_{t}, t$ in $T$, are uniformly bounded. Furthermore we assume the existence of a suitable probability measure $\mu$ on subsets of $T$. The nature of the measure $\mu$ is clear in the context of a problem, and it is generally chosen to be a "uniform" measure on $T$. Define the function $d$ on $Y \times Y$ by the formula 


$$
d^{2}(p, q)=\int|t(p)-t(q)|^{2} d \mu(t)
$$

LEMMA 1. The function $d$ is a metric on $Y$, and $\tau$ agrees with the topology induced by $d$.

Proof. The triangle inequality holds. For $p$ fixed $t(p)$ is a function in $L^{2}(T, \mu)$ since $|t(p)|$ is bounded, and the assertion that $d$ is a metric follows.

Next observe that a sequence of point $\left\{p_{n}\right\}$ in $Y$ tends to $p_{0}$ if and only if for each $t, \lim _{n}\left|t\left(p_{n}\right)-t\left(p_{0}\right)\right|=0$. Therefore the bounded convergence theorem assures that $\lim _{n} d\left(p_{n}, p_{0}\right)=0$ if and only if $\lim _{n} p_{n}=p_{0}$ with respect to the topology $\tau$. Therefore the metric topology of $d$ agrees with $\tau$.

We also wish to point out that the Cauchy-Schwarz inequality gives the important auxiliary inequality

$$
d(p, q) \geqq \int|t(p)-t(q)| d \mu(t) .
$$

The inequality (2), which will be strict unless $|t(p)-t(q)|$ is constant a.e., is often useful in identifying extremal configurations.

THEOREM 1. The metric space $(Y, d)$ may be isometrically embedded into $l^{2}$.

Proof. Perhaps the most direct proof follows from the observation that $(Y, d)$ embeds into $L^{2}(T, \mu)$, which in turn will embed into $l^{2}$. However we wish to base the proof on the following criterion of I. J. Schoenberg: A separable semimetric space $(S, \delta)$ embeds isometrically into $l^{2}$ if and only if for each finite set of points $\left\{p_{0}, p_{1}, \cdots, p_{n}\right\}$ in $S$, the quadratic form $\sum_{i j} \delta^{2}\left(p_{i}, p_{j}\right) x_{i} x_{j}$ is negative semidefinite on the plane $\sum_{i} x_{i}=0$.

To prove the theorem we observe that if $\left\{p_{0}, p_{1}, \cdots, p_{n}\right\}$ lie in $Y$, then for each $t$, the quadratic form $Q_{t}=\sum_{i j}\left|t\left(p_{i}\right)-t\left(p_{j}\right)\right|^{2} x_{i} x_{j}$ is negative semidefinite on the plane $\sum_{i} x_{i}=0$. Since $\mu$ is a positive measure, the form $Q=\int Q_{t} d \mu(t)$ shares this property. Hence $(Y, d)$ embeds isometrically into $l^{2}$. The space $(Y, d)$ is called the metric sum.

For convenience we shall write $Y=\sum Y_{t}$ to indicate a metric sum. The metric sum is clearly unique up to isometry. A central feature of the metric sum is its preservation of topological properties. This is both a strength and a weakness, since the metric sum applies only to mutually homeomorphic subsets of $l^{2}$. 
2. Some special embedding theorems, Let us consider the special situation where $Y$ consists of $n+1$ points, $T=\left\{t_{1}, t_{2}, \cdots, t_{s}\right\}$, and $Y_{t}$ lies in $E^{m}$ for each $t$. Define $\mu$ by $\mu\left(t_{i}\right)=\alpha_{i}^{2}$, where $\sum_{i} \alpha_{i}^{2}=1$. Here we may avoid Theorem 1 by means of direct construction. Set

$$
p_{i}^{\prime}=\left(\alpha_{1} t_{1}\left(p_{i}\right), \cdots, \alpha_{s} t_{s}\left(p_{i}\right)\right) .
$$

Note that $p_{i}^{\prime}$ lies in $E^{s m}$, and $\left|p_{i}^{\prime}-p_{j}^{\prime}\right|^{p}$ is in fact given by formula (1). This construction immediately proves the following special embedding theorem.

THEOREM 2. Let $Y$ be a set of cardinality $n+1$, where $n$ may be infinite, and let $T=\left\{t_{1}, t_{2}, \cdots, t_{s}\right\}$ be a finite set of homeomorphisms of $Y$ into the Euclidean space $E^{m}$. Then $\sum Y_{t}$ may be embedded into $E^{k}$ where $k=\min \{s m, n+1\}$.

As a further example let $Y=\{p(s): 0 \leqq s<1\}$ be a closed rectifiable curve of unit length, parametrized by arclength modulo one, which lies in $E^{m}$, and let $t$ lie in $[0,1)$. The mapping $p(s) \rightarrow$ $p(s+t)$ gives a natural homeomorphism. If we identify $t_{1}$ and $t_{2}$ with the induced homeomorphisms, and put $\mu\left(t_{1}\right)=\alpha^{2}, \mu\left(t_{2}\right)=\beta^{2}$, $\alpha^{2}+\beta^{2}=1$, then

$$
\sum Y_{t}=\left(\alpha p\left(s+t_{1}\right), \beta p\left(s+t_{2}\right)\right), \quad 0 \leqq s<1 .
$$

Clearly $\sum Y_{t}$ is a closed curve which lies in $E^{2 m}$. Since for each $t$, $p(s+t)$ is absolutely continuous, we may compute the length of $\sum Y_{t}$ in the usual manner and observe that it also is of unit length.

We state a theorem which contains the above example as a special case. We will not include a proof since the essential features of a proof in the case $d \mu(t)=d t$ are given in [1]. This proof can be easily modified to handle the more general $\mu$.

THEOREM 3. Let $Y=\{p(s): 0 \leqq s<1\}$ be a closed rectifiable curve in $l^{2}$ of unit length which is parametrized by arclength modulo one. If $\mu$ is a probability measure on $[0,1)$, then the metric sum $\sum Y_{t}$ over the homeomorphisms $t$ given by $t(p(s))=p(s+t)$ is again a curve of unit length.

Theorem 3, when combined with G. T. Sallee's chord theorem [8], is every effective in the investigation of metric inequalities for plane curves. Sallee's results often allow one to bypass a potentially fatal flaw of Theorem 3, namely that even though $Y$ may lie in $E^{2}, \sum Y_{t}$ seems trapped in a space of much higher dimension. A clear description of the general process is given in [1]. 
As indicated by Theorem 3, the topological properties of $Y$ can be an essential feature of a problem. However, in the remainder of this article we shall be primarily interested in metric $n$-tuples lying in $l^{2}$. The basic inequalities which we study can easily be extended to more general $Y$ by means of compactness arguments.

3. The circumsphere, If a pointset in $l^{2}$ lies on a Hilbert sphere of radius $R$, and $R$ is the least number for which this is true, then this sphere is called a circumsphere and $R$ is the circumradius. If the set is finite, we may take the Euclidean sphere of least dimension which contains the set (on its surface) as a circumsphere. For convenience we allow $R$ to be infinite, as in the case of three collinear points.

Lemma 2. Let $\left\{p_{0}, p_{1}, \cdots, p_{n}\right\}$ be points which are the vertices of $a$ nondegenerate simplex in $E^{n}$ and let $Q\left(x_{0}, x_{1}, \cdots, x_{n}\right)=$ $\sum_{i j}\left|p_{i}-p_{j}\right|{ }^{2} x_{i} x_{j}$. If $R$ is the circumradius of the points then $2 R^{2}$ equals the maximum of $Q$ subject to the condition $\sum_{i} x_{i}=1$.

Proof. Let the center of the circumsphere be the origin so that $\left|p_{i}\right|=R$ for each $i$. Using the fact that $\left|p_{i}-p_{j}\right|^{2}=2\left(R^{2}-p_{i} \cdot p_{j}\right)$, one easily establishes the identity

$$
Q\left(x_{0}, x_{1}, \cdots, x_{n}\right)=2 R^{2}-2\left|\sum_{i} x_{i} p_{i}\right|^{2} .
$$

It is seen immediately that $Q \leqq 2 R^{2}$ and this maximum is attained for the unique choice of $x_{0}, \cdots, x_{n}$ for which the affine sum $\sum_{i} x_{i} p_{i}$ is the origin. If the method of Lagrange multipliers is used to maximize $Q\left(x_{0}, \cdots, x_{n}\right)$ subject to $\sum_{i} x_{i}=1$, one obtains explicit formulas for the $x_{i}$.

Also, a simple continuity argument shows that Lemma 2 is valid even if $\left\{p_{0}, \cdots, p_{n}\right\}$ are the vertices of a degenerate simplex since $R$ is a continuous function of these points. Note that $R$ may or may not be infinite in the degenerate situation.

A straightforward argument (see [3, §3]) shows that for a compact set $K$ in $l^{2}, 2 R^{2}$ equals $\sup _{\eta} \iint|p-q|^{2} d \eta(p) d \eta(q)$ as $\eta$ varies over all signed Borel measures of total mass one concentrated on $K$.

THeOREM 4. Let $Y=\sum Y_{t}$ be a metric sum in $l^{2}$. Then

$$
R^{2} \leqq \int R_{t}^{2} d \mu(t)
$$

where $R$ and $R_{t}$ are the respective circumradii of $Y$ and $Y_{t}$. 
Proof. We will assume that $Y$ is a finite set $\left\{p_{0}, \cdots, p_{n}\right\}$. Now $2 R^{2}=\max Q=\max \int Q_{t} d \mu(t) \leqq \int \max Q_{t} d \mu(t)=2 \int R_{t}^{2} d \mu(t)$, with the side condition $\sum_{i} x_{i}=1$ understood to hold throughout. The extension of this proof for compact $Y$ is straightforward, and therefore is omitted.

THEOREM 5. For each $t$ let $Y_{t}$ be contained in a ball of radius R. Then $Y=\sum Y_{t}$ is contained in a ball of radius $R$.

Proof. Assume that $Y=\left\{p_{0}, p_{1}, \cdots, p_{n}\right\}$ is a finite set. For each $t$ choose a point $q_{t}$, not in $Y_{t}$, within distance $\varepsilon$ of the center of the ball containing $Y_{t}$. Let $Y_{t}^{\prime}=Y_{t} \cup\{q\}$ and form the metric sum $Y^{\prime}=\sum Y_{t}^{\prime}$. Clearly $Y^{\prime}$ may be viewed as the pointset $\left\{p_{0}, \cdots, p_{n}, q\right\}$ where $q$ is a point in $l^{2}$ whose existence is guaranteed by Theorem 1. For each $i,\left|q-p_{i}\right|^{2}=\int\left|q_{t}-t\left(p_{i}\right)\right|^{2} d \mu(t) \leqq(R+\varepsilon)^{2}$. It follows at once that $Y$ is contained in a closed ball radius $R$. We omit the easy extension of this argument to compact $Y$.

4. The distance between flats. Let $\left\{p_{0}, p_{1}, \cdots, p_{m}\right\}$ and $\left\{q_{0}, q_{1}, \cdots, q_{n}\right\}$ be pointsets in $l^{2}$ and let $Q$ be the quadratic form

$$
\sum\left|p_{i}-p_{j}\right|^{2} x_{i} x_{j}+\sum\left|p_{i}-q_{j}\right|^{2} x_{i} y_{j}+\sum\left|q_{i}-q_{j}\right|^{2} y_{i} y_{j} .
$$

Lemma 3. Let $h$ be the distance between the two flats generated by $\left\{p_{0}, p_{1}, \cdots, p_{m}\right\}$ and $\left\{q_{0}, q_{1}, \cdots, q_{n}\right\}$, respectively. Then

$$
h^{2}=\min |Q| \quad \text { subject to } \quad \sum_{i} x_{i}=1, \quad \sum_{i} y_{i}=-1 \text {. }
$$

Proof. If $\sum_{i} x_{i}=1$ and $\sum_{i} y_{i}=-1$, the following equation becomes an identity.

$$
-Q=\left|\sum_{i} x_{i} p_{i}+\sum_{i} y_{i} q_{i}\right|^{2} \text {. }
$$

The identity is easily established via the identity $2 p \cdot q=|p|^{2}+|q|^{2}-$ $|p-q|^{2}$ after "squaring out" the right hand side. We omit the details.

The special case of the identity (7) for three noncollinear points $\left\{p_{0}, p_{1}, q_{0}\right\}$ is known as Stewart's theorem. Here $-Q$ gives the squared length of the cevian from $q_{0}$ to the point $x_{0} p_{0}+x_{1} p_{1}$. Also, M. Klamkin has used versions of the identity (7) in a number of ingenius ways. His interpretation of (7) involves the notion of a moment of inertia. The article [6] gives an indication how this works.

Note that $|Q|$, subject to the side conditions, ranges over all 
possible values of $|p-q|^{2}$ where $p, q$ are arbitrary points on the flats generated by $\left\{p_{0}, \cdots, p_{m}\right\}$ and $\left\{q_{0}, \cdots, q_{n}\right\}$, respectively. This proves the lemma.

TheOREM 6. Let the pointsets $\left\{p_{0}, p_{1}, \cdots, p_{m}\right\}$ and $\left\{q_{0}, q_{1}, \cdots, q_{n}\right\}$ be the respective metric sums of the pointsets $\left\{t\left(p_{0}\right), t\left(p_{1}\right), \cdots, t\left(p_{m}\right)\right\}$, $\left\{t\left(q_{0}\right), t\left(q_{1}\right), \cdots, t\left(q_{n}\right)\right\}, t$ in $T$. Then if $h_{t}$ is the distance between the corresponding flats, we have

$$
h^{2} \geqq \int h_{t}^{2} d \mu(t)
$$

Proof. Subject to $\sum_{i} x_{i}=1, \sum_{i} y_{i}=-1$, it follows that

$$
h^{2}=\min |Q|=\min (-Q) \geqq \int \min \left(-Q_{t}\right) d \mu(t)=\int h_{t}^{2} d \mu(t) .
$$

Here

$Q_{t}=\sum\left|t\left(p_{i}\right)-t\left(p_{j}\right)\right|^{2} x_{i} x_{j}+\sum\left|t\left(p_{i}\right)-t\left(q_{j}\right)\right|^{2} x_{i} y_{j}+\sum\left|t\left(q_{i}\right)-t\left(q_{j}\right)\right|^{2} y_{i} y_{j}$.

Next we prove a result which is known when suitably reinterpreted. However, we give a proof based on Theorem 6 .

CoROLlaRY 1. Let $V_{t}$ be the volume of the simplex whose vertices are $\left\{t\left(p_{0}\right), \cdots, t\left(p_{n}\right)\right\}$ for $t$ in $\left\{t_{1}, t_{2}, \cdots, t_{s}\right\}$, and let $V$ be the volume of $\Sigma Y_{t}$. Then if $\mu(t)=1 / s$ for each $t$,

$$
V^{s} \geqq \prod_{t} V_{t} \text {. }
$$

Proof. Clearly $n ! V=h_{n} h_{n-1} \cdots h_{1}$ where $h_{i}$ is the altitude of an $i$-dimensional simplex. We may use Theorem 6 to assert

$$
\prod_{i=1}^{n} h_{i}^{2} \geqq \prod_{i=1}^{n}\left[\frac{1}{s} \sum_{j=1}^{s} h_{i j}^{2}\right],
$$

where $h_{i j}$ is the altitude corresponding to $h_{i}$ in the simplex whose vertices are $\left\{t_{j}(p)\right\}$. To apply Theorem 6 we let $\left\{p_{0}, \cdots, p_{i-1}\right\}$ be the vertices of the base simplex with $q_{0}=p_{i}$. The altitude $h_{i}$ is measured from $q_{0}$ to the flat determined by $\left\{p_{0}, \cdots, p_{i-1}\right\}$.

After applying the usual arithmetic-geometric mean inequality of each factor on the right side of (10) the inequality (9) follows almost at once. We will discuss (9) further in $\S 6$.

5. The medians of a simplex. If $Y=\left\{p_{0}, p_{1}, \cdots, p_{n}\right\}$ is the vertex set of an arbitrary simplex, then the median distance $m$ from $p_{n}$ is $\left|p_{n}-1 / n \sum_{i=0}^{n-1} p_{i}\right|$. If $Y$ is a metric sum $\sum Y_{t}$, then let 
$m_{t}$ be the corresponding median distance for the set $Y_{t}=$ $\left\{t\left(p_{0}\right), \cdots, t\left(p_{n}\right)\right\}$.

THEOREM 7. The median distance of a metric sum satisfies

$$
m^{2}=\int m_{t}^{2} d \mu(t)
$$

Proof. Set $q_{0}=p_{n}, y_{0}=-1$, and $x_{i}=1 / n$ for $i=1, \cdots, n-1$ in the identity (7), and note that $m^{2}=-Q$ while $m_{t}^{2}=-Q_{t}$. Since $Q=\int Q_{t} d \mu(t)$, the theorem is true. A practically identical proof shows that (11) holds for the "generalized medians" investigated in the article [2].

6. Positive definite matrices. For convenience let $d_{i j}=\left|p_{i}-p_{j}\right|$, $0 \leqq i, j \leqq n$. The substitution $x_{0}=-\left(\sum_{i=1}^{n} x_{i}\right)$ shows that the quadratic form $\sum d_{i j}^{2} x_{i} x_{j}$ is negative semidefinite on plane $\sum_{i=0}^{n} x_{i}=0$ if and only if the form $\sum_{i, j=1}^{n}\left(d_{0 j}^{2}+d_{0 j}^{2}-d_{i j}^{2}\right) x_{i} x_{j}$ is positive semidefinite. Likewise, if $x^{T} A x$ is a positive semidefinite form, there are points $p_{0}, p_{1}, \cdots, p_{n}$ such that $a_{i j}=2\left(p_{0}-p_{i}\right) \cdot\left(p_{0}-p_{j}\right)=d_{c i}^{2}+d_{0 j}^{2}-$ $d_{i j}^{2}, 1 \leqq i, j \leqq n$. This follows from the matrix equation $A=B^{T} B$ which characterizes positive semidefinite matrices. These observations lie at the heart of the Menger-Schoenberg embedding theory, and a careful investigation of these ideas from the point of view of classical matrix theory is given in the book [4].

Since the square of the volume of the simplex with vertices $\left\{p_{0}, p_{1}, \cdots, p_{n}\right\}$ is propositional to $\operatorname{det}\left(a_{i j}\right)$, a moments thought shows that Corollary 1 is equivalent to the assertion that if $A_{1}, \cdots, A_{s}$ are positive definite $n \times n$ matrices, then

$$
\operatorname{det}\left(\frac{1}{s} \sum_{i} A_{i}\right) \geqq\left(\operatorname{det} \prod_{i} A_{i}\right)^{1 / s} .
$$

This inequality is well-known (see [7]). Professor Narasimhan points out that the result for $s=2$ follows at once upon simultaneously diagonalizing $A_{1}$ and $A_{2}$. The full inequality (12) can then be deduced by a suitable induction process.

7. An example. In conclusion we will consider an illustrative problem which yields easily to the metric sum approach: Suppose four points $\left\{q_{0}, q_{1}, q_{2}, q_{3}\right\}$ lie on a unit sphere in $E^{3}$. Show that the sum of the four altitudes of the simplex they determine is maximal precisely when the simplex is regular.

Solution. Let $T$ be the 24 distinct homeomorphisms of a 
discrete set $\left\{p_{0}, p_{1}, p_{2}, p_{3}\right\}$ to the $\left\{q_{i}\right\}$. Set $\mu(t)=1 / 24$ for each $t$ in $T$, and form the metric sum. Thus for any $k \neq l,\left|p_{k}-p_{l}\right|^{2}=$ $1 / 24 \sum\left|t\left(p_{k}\right)-t\left(p_{l}\right)\right|^{2}=1 / 12 \sum_{i, j}\left|q_{i}-q_{j}\right|^{2}$.

The inequality (6) says that the points $\left\{p_{i}\right\}$ lie on a sphere in $E^{3}$ of radius $R \leqq 1$. If $h_{i}$ is the altitude from $q_{i}$ and $h_{i}^{\prime}$ is the altitude from $p_{i}$, the inequality (8) reduces to $h_{i}^{\prime 2} \geqq 1 / 4\left[h_{0}^{2}+h_{1}^{2}+h_{2}^{2}+h_{3}^{2}\right]$ which implies $h_{i}^{\prime} \geqq 1 / 4\left[h_{0}+h_{1}+h_{2}+h_{3}\right]$.

Thus we have shown that given any simplex whose vertices lie on a unit sphere, there is a regular simplex whose vertices lie on a sphere of radius not exceeding one, and whose altitude sum is as large as that of the original simplex. Therefore the regular simplex is extremal.

A somewhat more careful investigation is needed to show that the regular simplex is the unique extremal configuration. If the inequality (6) is not strict, then the values $x_{i}$ which minimize the quadratic form (5) must be invariant under all permutation and hence must all equal $1 / 4$. This says that the center of the sphere is the centroid of the points. Similar reasoning shows that $x_{0}=x_{1}=$ $x_{2}=1 / 3, y_{0}=-1$ must always minimize the form $-Q$ in (7). This means that the altitude are also medians. The three medians intersect at the center of the sphere since the center is the centroid.

Suppose the points $\left\{p_{i}\right\}$ form an extremal configuration. The altitude from $p_{0}$ passes through the center of the circumsphere and strikes the plane containing $\left\{p_{1}, p_{2}, p_{3}\right\}$ at the centroid of these three points. This centroid will also be the center of the small circle on which the three points lie. If three points lie on a circle, their centroid is the center if and only if they are the vertices of an equilateral triangle. Since this argument works at any vertex, the $\left\{p_{i}\right\}$ must be the vertices of a regular simplex.

The fact that all four altitudes are equal in an extremal configuration, taken alone, would only imply that the extremal simplex is isoceles. See [5, Chap. 9] for an account of isoceles tetrahedra.

\section{REFERENCES}

1. R. Alexander, Metric embedding techniques applied to geometric inequalities, The geometry of metric and linear spaces, Ed. L. M. Kelly, (Springer-Verlag, 1975), $57-65$.

2. The width and diameter of a simplex, Geometriae Dedicata, 6 (1977), 87-94.

3. R. Alexander and K. B. Stolarsky, Extremal problems of distance geometry related to energy integrals, Trans. Amer. Math. Soc., 193 (1974), 1-31.

4. L. M. Blumenthal, Theory and Applications of Distance Geometry, Clarendon Press, Oxford, 1953.

5. R. Honsberger, Mathematical Gems II, The Mathematical Association of America, 1976. 
6. M. S. Klamkin, Geometric inequalities via the polar moment of inertia, Math. Mag., 48 (1975), 44-46.

7. M. Marcus and H. Minc, A Survey of Matrix Theory and Matrix Inequalities, Allyn and Bacon, Boston, 1964.

8. G. T. Sallee, Stretching chords of space curves, Geometriae Dedicata, 2 (1974), 311-317.

9. I. J. Schoenberg, Remarks to Maurice Fréchet's article "Sur la définition axiomatique d'une classe d'espaces vectoriels distanciés applicables vectoriellment sur l'espace de Hilbert," Ann. of Math., 36 (1935), 724-732.

Received March 5, 1979.

UNIVERSITY OF ILLINOIS

URBANA, IL 61801 



\section{PACIFIC JOURNAL OF MATHEMATICS}

\section{EDITORS}

DONALD BABBITT (Managing Editor)

University of California

Los Angeles, California 90024

HUgo RossI

University of Utah

Salt Lake City, UT 84112

C. C. MOORE and ANDREW OGG

University of California

Berkeley, CA 94720

\section{J. DUGUNDJI}

Department of Mathematics University of Southern California Los Angeles, California 90007

R. Finn and J. Milgram Stanford University Stanford, California 94305

\section{ASSOCIATE EDITORS}

E. F. BECKENBACH

B. H. NEUMANN

F. WOLF

K. YoshidA

\section{SUPPORTING INSTITUTIONS}

UNIVERSITY OF BRITISH COLUMBIA CALIFORNIA INSTITUTE OF TECHNOLOGY UNIVERSITY OF CALIFORNIA MONTANA STATE UNIVERSITY UNIVERSITY OF NEVADA, RENO NEW MEXICO STATE UNIVERSITY OREGON STATE UNIVERSITY UNIVERSITY OF OREGON
UNIVERSITY OF SOUTHERN CALIFORNIA STANFORD UNIVERSITY UNIVERSITY OF HAWAII UNIVERSITY OF TOKYO UNIVERSITY OF UTAH WASHINGTON STATE UNIVERSITY UNIVERSITY OF WASHINGTON 


\section{Pacific Journal of Mathematics}

Vol. 85, No. $1 \quad$ September, 1979

Ralph Alexander, Metric averaging in Euclidean and Hilbert spaces...... 1

B. Aupetit, Une généralisation du théorème de Gleason-Kahane-Żelazko

pour les algèbres de Banach..............................

Lung O. Chung, Jiang Luh and Anthony N. Richoux, Derivations and

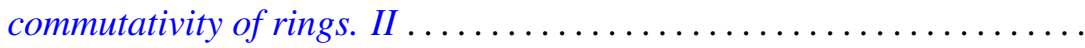

Lynn Harry Erbe, Integral comparison theorems for third order linear

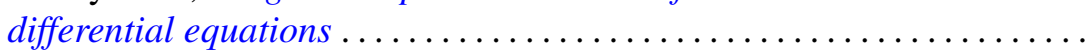

Robert William Gilmer, Jr. and Raymond Heitmann, The group of units of a

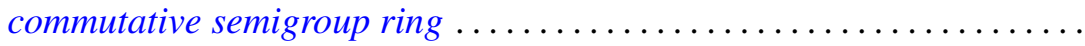

George Grätzer, Craig Robert Platt and George William Sands, Embedding lattices into lattices of ideals ...........................

Raymond D. Holmes and Anthony Charles Thompson, $n$-dimensional area and content in Minkowski spaces ....................... 77

Harvey Bayard Keynes and M. Sears, Modelling expansion in real flows....

Taw Pin Lim, Some classes of rings with involution satisfying the standard

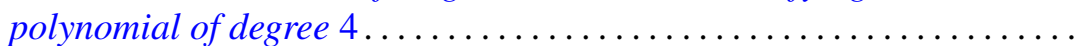

Garr S. Lystad and Albert Robert Stralka, Semilattices having bialgebraic congruence lattices ................................... 131

Theodore Mitchell, Invariant means and analytic actions . . . . . . . . . 145

Daniel M. Oberlin, Translation-invariant operators of weak type ........ 155

Raymond Moos Redheffer and Wolfgang V. Walter, Inequalities involving

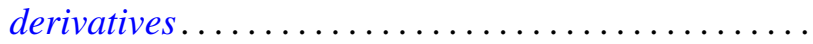

Eric Schechter, Stability conditions for nonlinear products and semigroups ................................

Jan Søreng, Symmetric shift registers ........................ 201

Toshiji Terada, On spaces whose Stone-Čech compactification is $\mathrm{Oz} \ldots \ldots .231$

Richard Vrem, Harmonic analysis on compact hypergroups ... 